\title{
Ueber die septischen Amputationen.
}

Von Stabsarzt Dr. H. H an t s ch e r (Konstantinopel), z. Z. in Berlin.

Wir verfolgen zwei Hauptziele: 1. die Amputationswunden sehnell zum Heilen zu bringen, und 2. für die Prothesen gut geeignete Stumpfformen herzustellen, ohne sehlcehte Kallus- und Narbenbildung.

Dic Art und Weise der Operation ist von großer Bedeutung für die Disuer des ehirurgischen Eingriffs.

Bei septisehen Amputationen darf man niemals einen Lappen herstellen, um das Ende cies Knochens zu verschließen, da ein solcher nur dazu dient, die Zeit der $\mathrm{H}$ ilung der Wunde zu verzögern, indem er mehrere $T_{a}$ schen für eine Eite ung bildet, ihr eine größere Oberfläche bietct und schließlich eine große, sehr unregelmäßige, liarte und rote Narbenbildung, dic dic Neigung hat, zu vereitern, kleine, oberflächliche Wunden bietet und sich direkt auf einen sehleehtgebildeten, hypertrophisclien Kallus stützt, der dauernde Sehmę̧zen, die die Kranken selbst verhindern, die Prothesen zu tragen, verursacht. Man darf niemals das Messer senkrecht zur Richtung der Wunde gebrauchen. Nachdem man die Haut abgesehnitten hat, kann man sie hochziehen, um die Muskeln abzuschneiden. Die Elastizität des subkutanen Zellgewebes muß genügen, um einen kleinen Längenuntersehied zu erziclen. Wir brauchen keinen sehr großen Hautlappen, weil in der Zeit, die die Heilung der Wunde in Anspruch nchmen wird, dic Muskeln sich weiter zusammenzichen als die Haut.

Während man die Muskeln absehneidet, muß man gut darauf aehten,

1) M. Kl. 1916 Nr. 34. 
bei einer guten Narkose, daß sie völlig entspannt sind. Wir wissen bereits, daß die Muskeln sich verschieden stark zusammenziehen; wenn die Entspannung der Muskeln nicht vollständig ist, so wird dieser Unterschied größer, und es bildet sich eine sehr unregelmäßige Oberfläche, die der längeren Dauer der Eiterung günstig ist. Daher stammt das Verbot, große Amputationen unter Lokalbetäubung vorzunehmen.

Man muß bei septischen Amputationen darauf bedacht sein, die Knochen kürzer zu schneiden $z l s$ bei aseptischen. Es ist bisher reine mechanische Möglichkeit bekannt, die die stets weiter fortschreitende Retraktion. der Muskeln zu hindern vermöchte, auch die bisher oft angewandte Extension hat keine er. sprießlichen Resultate geliefert. Folg. lich kommt es auf die Kürzung des Knochens an, jedoch sind die bislang angewandten Kompressions- und Platten - Retraktionsapparate nicht ausreichend, um zum gewünschten Ziele zu kommen. Sowohl mittels des Zuges durch ein Handtuch, als auch den Retrakteur (z. B. Retrakteur von Roux) können wir von a $a^{\prime}$ (Fig. 1) nach A A'. Man muß die Weichteile 'zurückschieben in Richtung $\mathrm{BE}$ und $\mathrm{B}^{\prime} \mathrm{E}^{\prime}$, damit man bei $\mathrm{E} \mathrm{E}^{\prime}$ auf den Knochen kommt. Nun haben wir einen Apparat konstruiert, um zu diesem Ziel zu kommen, und ihm, um recht viel Platz zu gewinnen, schräggestellte, gebogene Seitenflächen $\mathrm{H} \mathrm{H}^{\prime}$ gegeben. soda $\boldsymbol{B}$ die Knochenpunkte $E E^{\prime}$ tiefer liegen.

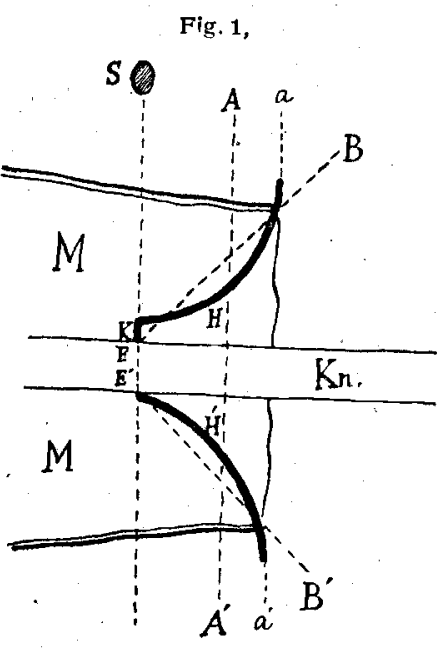
widerstandsfähigen Knochengewebes besteht. Ist der Eiter einmal mit dem periostlosen Knochen in Berührung gekommen, so muß man an die Gefahr seines Absterbens denken und einige Zeit warten (zwei bis vier Monate), bis der Prozeß an dieser Stelle zur Sequestrierung geführt hat, um dann die a bgestorbenen Knochenteile zu exstirpieren. Wir
Fig. 3.

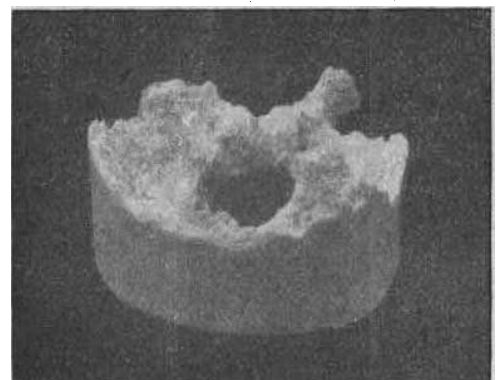
hatten Gelegenheit, mehrere solche Fälle zur beobachten; Fistelbildungen deuerten mehrere Monate lang an infolge von Sequestern am Ende des amputierten Knochens. Die Sequester haben zuweilen die Form einer Krone (Fig. 3), die im allgemeinen jedoch nicht vollständig ist, wenn auch manchmal nur ein kleiner Teil abgestorben ist und fehlt. Am unangenehmsten ist es, und gleichzeitig am häufigsten kommt es vor, daß man diesen abgestorbenen Teil garnicht findet oder ihn in der Wunde liegen läßt. Das sind.dann die kleinen, verstreuten, im Kallus liegenden Teilchen, Eiterherde, die den Kallus verunreinigen und ein krankes, hypertrophisches, wenig widerstandsfähiges Gewebe entstehen lassen, das kleine Knochen. teile mehr oder weniger resorbiert einschließt.
Bei $\mathrm{E}$ ist Platz genug, die Säge anzusetzen. So ähnelt dieser Apparăt etwa einer Wasserrinne, die einen ebenen Grund und konvexe, nach oben oval abgerundete Seitenflachen hat. Der Apparat besteht also aus zwei Teilen $A$ und $B$ (Fig. 2), die durch das in einer Ebene gegen-

Fig. 2.

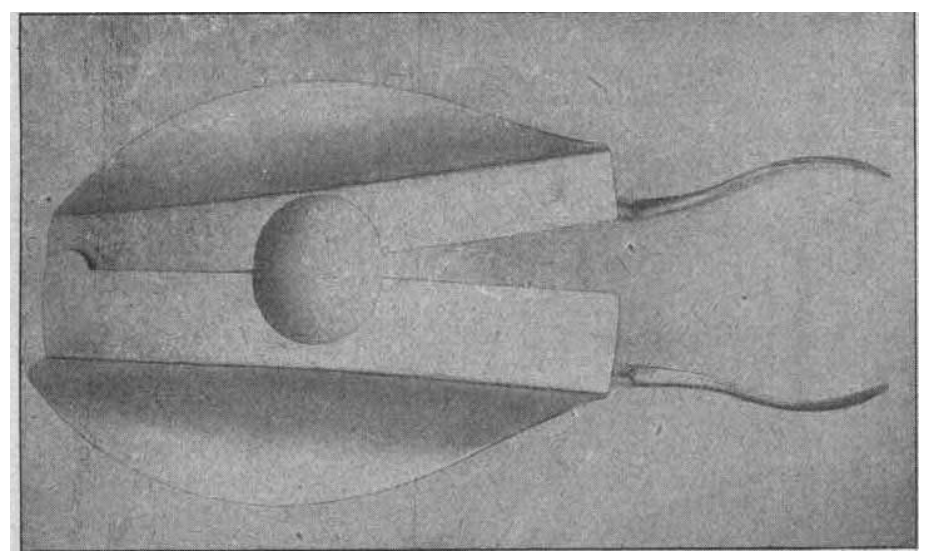

einander verschiebliche Gelenk (c) verbunden sind und von denen jeder das Gelenk (c) umfaßt, indem man die Handgriffe auseinandernimmt, wodurch die Oeffnung sich erweitern läßt. Die beiden Hälften schließen dann den Knochen in der Oeffnung $\mathrm{F}$ ein. Mag es sich beispielsweise um den Femur handeln. Man drückt stets mittels der,Griffe $\mathrm{E} \mathrm{E}^{\prime}$ die Lochränder fest auf dem Knochen zusammen, um sich das bereits vorher abgelöste Periost sowie die Weichteile nicht entgehen zu lassen, und zieht den Apparat so weit als möglich zurück. Dann sägt man mit einer Bogensäge, deren Sehne etwa 5-8 $\mathrm{mm}$ breit ist und in der Rinne $(\mathrm{K})$ (Fig. 1) angesetzt wird, den Knochen durch; es muß hier besondere Sorgfalt auf die genau zum Knochen vertikale Sägerichtung gelegt werden, d. h. die oberen Muskelteile liegen zwischen Bogen und Sehne der Säge. Ist der Knochen einmal durchsägt, so hat man nur noch das Periost genügend weit und gut vorbereitet um das Knochenende herumzulegen und die Wunde zu bedecken. Man darf niemals einen. Drain oder Mullstreifen in der Richtung auf den Knochen anlegen, der den Knochen erreicht. Wenn man aber später den Eiter aus einer schon vorgebildeten Tasche herausfließen sieht, so legt man dann einen Kapillardrain ein: den neuen Kapillardrain aus Kautschuk ${ }^{\mathbf{1}}$ ), durch den der Eiter abfließen kann, obgleich er von weichen Muskelteilen umgeben und ein. geengt ist. Es ist allgemein bekannt, $\mathrm{da} \beta$ das gefährlichste und (fast einzige) ausschlaggebende Moment für das Offenbleiben der Amputationswunden über Monate hinaus in der Bildung eines nekrotischen, wenig

1) D. m. W. 1916 Nr. 36. 